\title{
A model of cerebral aspergillosis in non-immunosuppressed nursing rats
}

\author{
Stefan Zimmerli $\cdot$ Urspeter Knecht $\cdot$ Stephen L. Leib
}

Received: 9 March 2007 / Revised: 4 June 2007 / Accepted: 8 June 2007 / Published online: 30 June 2007

(C) Springer-Verlag 2007

\begin{abstract}
Central nervous system aspergillosis is an often fatal complication of invasive Aspergillus infection. Relevant disease models are needed to study the pathophysiology of cerebral aspergillosis and to develop novel therapeutic approaches. This study presents a model of central nervous system aspergillosis that mimics important aspects of human disease. Eleven-day-old non-immunosuppressed male Wistar rats were infected by an intracisternal injection of $10 \mu \mathrm{l}$ of a conidial suspension of Aspergillus fumigatus. An inoculum of $7.18 \log _{10}$ colony-forming units (CFU) consistently produced cerebral infection and resulted in death of all animals $(n=25)$ within 3-10 days. Median survival time was 3 days. Histomorphologically, all animals developed intracerebral abscesses (2-26 per brain) containing abundant fungal hyphae and neutrophils. Fungal culture of cortical homogenates yielded maximal growth on day 3 after infection (5.4 $\log _{10} \mathrm{CFU} / \mathrm{g}, n=15$ ) that declined over time. Galactomannan concentrations in cortical homogenates, assessed as an index for hyphal burden, peaked on days 3-5. Fungal infection spread to peripheral organs in $83 \%$ of animals. Fungal burden in lung, liver, spleen and kidney was two orders of magnitude lower than in the brain. The successful establishment of a model of cerebral aspergillosis in a non-immunosuppressed host provides the opportunity to investigate mechanisms of disease and to develop novel treatment regimens for this commonly fatal infection.
\end{abstract}

This work was supported in part by an unrestricted educational grant from Pfizer AG, Switzerland.

S. Zimmerli $(\bowtie) \cdot$ U. Knecht $\cdot$ S. L. Leib

Institute for Infectious Diseases, University of Bern,

Friedbuehlstr, 51, P.O. Box 61, 3010 Bern, Switzerland

e-mail: stefan.zimmerli@ifik.unibe.ch
Keywords Aspergillosis · Central nervous system · Animal model $\cdot$ Rat $\cdot$ Galactomannan

\section{Introduction}

Invasive aspergillosis is a major cause of illness and death in immunocompromised subjects, including patients with hematologic cancers and recipients of bone marrow and solid-organ transplants. Risk factors are prolonged neutropenia, graft-versus-host disease, high-dose corticosteroid treatment and other immunosuppressive regimens. Cerebral aspergillosis occurs at a frequency of $14-42 \%$ in patients with acute leukemia or allogeneic stem-cell transplantation making it the most common organ manifestation of hematogenous dissemination from primary foci, usually in the lungs [16, 24]. Alternatively, cerebral aspergillosis can arise by direct invasion from adjacent infectious foci e.g., sinusitis. The pathophysiology of cerebral aspergillosis is incompletely understood. Ischemic and hemorrhagic infarcts are thought to result from arteriolitis and arteritis with fungal thrombosis and obliteration of the vessel lumen. Cerebral abscesses characterized by necrotic parenchyma, a paucity of inflammatory cells and fungal hyphae are commonly found, but their pathogenesis has remained largely unknown [30]. Until recently, cerebral aspergillosis was considered to be universally lethal $[16,24,30]$. Recently introduced antifungal drugs with enhanced central nervous system penetration and novel mechanisms of action offer promising treatment options. Indeed, the combination of voriconazole and neurosurgery resulted in complete or partial responses in 28 of 81 patients with central nervous system (CNS) aspergillosis [27]. The role of posaconazole and the echinocandins in the treatment of cerebral aspergillosis remains to be defined. Relevant disease models are needed 
to study the pathophysiology of cerebral aspergillosis and to develop improved treatment regimens.

A recently established model of aspergillosis in immunosuppressed mice uses direct intracerebral injection of conidia to induce cerebral abscesses [3]. This model has been used successfully for studies on drug efficacy and disease progression $[4,5,6,8,14,15,29]$. However, persistent pancytopenia induced by the high level of immunosuppression necessary to establish infection and the significant systemic disease that likely contributes to mortality may be considered as the disadvantages of this model.

Building on our experience with experimental CNS infection in rats, we have established a model of cerebral aspergillosis in non-immunosuppressed nursing rats that mimics important aspects of human disease [1, 10, 22, 23, 28]. To allow sufficient time to study disease progression and potential treatment effects, CNS infection in untreated animals was intended to be universally and reproducibly lethal within several days.

\section{Materials and methods}

\section{Fungal inoculum}

The strain of Aspergillus fumigatus used for infection was isolated from a patient with invasive pulmonary aspergillosis. The isolate was cultivated on Sabouraud's agar plates at $37^{\circ} \mathrm{C}$ for 3 days [31]. Conidia were harvested by washing the plates with phosphate-buffered saline (PBS) supplemented with $0.05 \%$ Tween 80 and filtering the resulting suspension through a BD Falcon $40 \mu$ m nylon cell strainer (BD Biosciences, Bedford, MA, USA) to eliminate hyphal fragments and conidial clumps. The concentration of the resulting stock suspension of predominately single spores was repeatedly verified by culturing serial dilutions on Sabouraud's agar plates. Colony-forming units (CFU) were counted after $20 \mathrm{~h}$ at $37^{\circ} \mathrm{C}$. Three days before infection, the stock suspension (stored at $4^{\circ} \mathrm{C}$ ) was diluted with $0.05 \%$ Tween 80 in PBS to the desired concentration, which was verified by culture. In addition, the fungal titer of the inoculum was routinely confirmed by quantitative culture on the day of infection.

\section{Rats}

Nursing Wistar rat pups (Charles River Laboratories, Sulzfeld, Germany) were kept in groups of four to eight with their dam for 5 days before the experiments. Water and food were provided ad libitum during a 12-h day-night cycle. The animal studies were approved by the Animal Care and Experimentation Committee of the Kanton of Bern, Switzerland, and followed National Institutes of
Health guidelines for the performance of animal experiments.

To produce cerebral Aspergillus infection

No immunosuppressive drugs were used. The relative immaturity of the immune system of infant rats permits establishment of invasive aspergillosis without iatrogenic immunosuppression. To produce cerebral Aspergillus infection, the inoculum was injected into the cisterna magna of the rats. The procedure that does not produce structural brain damage was well tolerated by all animals and no deaths were observed during the first $12 \mathrm{~h}$ after injection. On postnatal day 11 , when the rats $(n=97)$ weighed $26.5 \pm 3.9 \mathrm{~g}$, they were infected by a direct intracisternal injection, with a 32-gauge needle of $10 \mu \mathrm{l}$ of saline containing Aspergillus conidia [1, 10, 22, 23, 28]. In preliminary experiments, the rats $(n=12)$ were given inocula of 5.0, 6.0 and $7.0 \log _{10} \mathrm{CFU}$ ( $n=4$ per group). One animal of each group was killed on day 4 for quantitative cerebral fungal culture (resulting in 3.2, 3.4 and $4.6 \log _{10} \mathrm{CFU} / \mathrm{g}$, respectively) and three out of four were observed for survival to day 7 after infection when the experiment was censored. The median survival of animals infected with inocula $\leq 7.0 \log _{10} \mathrm{CFU}$ was 7 days (range 3-7). For an inoculum of $5.0 \log _{10}$ CFU survival was $100 \%$.

In the dose finding studies, four different inocula of A. fumigatus conidia were injected: $7.18 \log _{10} \mathrm{CFU}(n=25)$, $7.3 \log _{10} \mathrm{CFU} \quad(n=9), \quad 7.48 \log _{10} \mathrm{CFU} \quad(n=11) \quad$ and $7.70 \log _{10} \mathrm{CFU}(n=8)$. In a pilot experiment, corticosteroids were administered immediately after infection in an attempt to decrease the mortality associated with higher inocula $(n=18)$. However, dexamethasone administered at a dose of $0.5 \mathrm{mg} / \mathrm{kg}$ after inoculation of $7.3 \log _{10}$ CFU $(n=8)$ or at $4 \mathrm{mg} / \mathrm{kg}$ following infection with $7.48 \log _{10}$ CFU $(n=10)$ was associated with $100 \%$ mortality within $24 \mathrm{~h}$. Because of the precipitous mortality associated with corticosteroid use, we decided to limit our study to animals without iatrogenic immunomodulation.

In an additional group of 14 rats, the kinetics of intracerebral fungal replication during the first 4 days after infection was studied.

The size of the inoculum used for infection, calculated to contain $7.18 \log _{10} \mathrm{CFU} / 10 \mu \mathrm{l}$ was confirmed by culture of serial dilution and yielded a growth of $7.17 \pm$ $6.54 \log _{10} \mathrm{CFU} / 10 \mu \mathrm{l}(n=3)$. Uninfected control animals were injected with $10 \mu \mathrm{l}$ of sterile saline.

The intracisternal injection was performed by the same experimenter in all animals. The procedure failed to produce an infection in 14/111 rats that were excluded from evaluation. These animals survived to day 11 without clinical signs of infection; they were culture-negative in all organs studied: brain, lung, liver, kidney and spleen, and 
there was no histopathological evidence of infection in the brain. Failure to produce infection may result from subcutaneous deposition of the inoculum. We observed failures as isolated occurrences, except in one underperforming experiment where 7 of 14 animals were not infected. Infection was not produced in 2 of 6 rats with an inoculum of $7.0 \log _{10}$ CFU, 9 of 48 with an inoculum of $7.18 \log _{10}$ CFU (7 of which in one experiment), 1 of 10 with an inoculum of $7.3 \log _{10} \mathrm{CFU}$ and 2 of 13 with an inoculum of $7.48 \log _{10}$ CFU. In the absence of a non-invasive method to discriminate infected from non-infected animals, we retrospectively identified failure of infection by a comprehensive analysis of all parameters assessed including disease-free survival, weight gain, quantitative organ culture, galactomannan determination and histopathology.

After infection, all animals were assessed clinically twice daily using a scoring system with a range of 1-5 based on activity, time to return from a supine to an upright position and alertness (5-no signs of disease; 4-slightly reduced activity, animal turns upright in $<5 \mathrm{~s}$; 3 -reduced activity, animal turns upright in $>5$ and $<30 \mathrm{~s} ; 2$ - loss of capacity for coordinated and purposeful activity, animal fails to turn upright; 1 -coma). Animals with a score of $\leq 2$ were killed for ethical reasons with an overdose of pentobarbital $(100 \mathrm{mg} / \mathrm{kg}$ intraperitoneally) and subsequently perfused via the right cardiac ventricle with ice cold PBS. In survival studies, the day of death was assigned to the day the animals were killed for ethical reasons $(n=41)$; for animals found dead $(n=21)$, death was assigned to the same day.

\section{Fungal cultures}

One sagital half brain, the left lung, one kidney, the liver, and the spleen were weighed, diluted 1:1 (w:v) in PBS, homogenized in a tissue homogenizer, serially diluted $10^{-2}$ to $10^{-4}$ in PBS, and cultured on Sabouraud's agar plates. $\mathrm{CFU}$ were counted after $20 \mathrm{~h}$ at $37^{\circ} \mathrm{C}$. Plates with no growth after 5 days were considered sterile. The detection limit was $200 \mathrm{CFU} / \mathrm{g}$ of tissue. To better characterize the early stages of infection, animals infected with $7.18 \log _{10}$ $\mathrm{CFU}$ of A. fumigatus conidia were sacrificed at 1, 2, 3, and 4 days after infection ( 3 to 4 animals per time point) and brain homogenates were processed for fungal culture.

\section{Histopathologic examination}

The sagittal half brains not used for culture were fixed for $12 \mathrm{~h}$ in $4 \%$ formaldehyde in PBS and paraffin embedded. Coronal sections obtained from the frontal, middle and dorsal brain regions were stained with Grocott's modified Gomori and periodic acid-Schiff stain.
Galactomannan determination

Galactomannan concentration was assessed in brain homogenates diluted in 1:40 in PBS according to the manufacturer's instruction (Platelia Aspergillus test, Bio-Rad Laboratories AG, Reinach, Switzerland). Concurrently measured internal controls and calibrators were used to determine the galactomannan index.

Statistical analysis

Statistical analyses were performed using GraphPad Prism (GraphPad Software Inc., San Diego, USA). Normally distributed variables (weight) are presented as mean \pm SD and were compared using one-way ANOVA followed by the unpaired student's $t$ test. Variables that were not normally distributed (CFU) were compared by the Kruskal-Wallis test. When the latter yielded a statistically significant value $(P<0.05)$, pair-wise comparison was done using the twotailed non-parametric Mann-Whitney $U$ test. Survival curves were analyzed by Kaplan-Meier analysis and compared by logrank test.

\section{Results}

Intracisternal infection of infant rats: dose-finding studies

Dose-finding studies with inocula ranging from 7.18 to $7.70 \log _{10}$ CFU of A. fumigatus were performed. The size of the inoculum showed a dose response with respect to median survival, which was 2 (range 1-2), 2 (1-6) and 3 (3-10) days for inocula of $7.70(n=8), 7.48(n=11)$ and $7.18 \log _{10}$ CFU $(n=25)$ of A. fumigatus, respectively (Fig. 1a). Median survival of rats infected with an inoculum of $7.3 \log _{10} \mathrm{CFU}(n=9)$ was $3(3-11)$ days (results not shown).

Figure $1 \mathrm{~b}$ shows the comparison of inoculum size and cerebral fungal burden in animals experiencing mortality in the first 3 days after infection. The number of Aspergillus $\mathrm{CFU}$ recovered from cortical homogenates was proportional to the size of the inoculum. It was $5.58 \log _{10}(4.48$ 6.64) for an inoculum of $7.18 \log _{10} \mathrm{CFU}, 6.07 \log _{10}$ (4.956.34) for an inoculum of $7.48 \log _{10}$, and 6.28 (5.51-6.45) for an inoculum of $7.70 \log _{10}$. Fungal recovery for an inoculum of $7.18 \log _{10}$ CFU was significantly lower than for the higher inocula $(P=0.014)$. Thus, the number of conidia in the inoculum used for infection determines both, cerebral fungal burden and mortality. On the basis of this result, we thought of balancing the severity of disease and mortality by choosing an inoculum size of $7.18 \log _{10} \mathrm{CFU}$ of $A$. fumigatus for further experiments. 


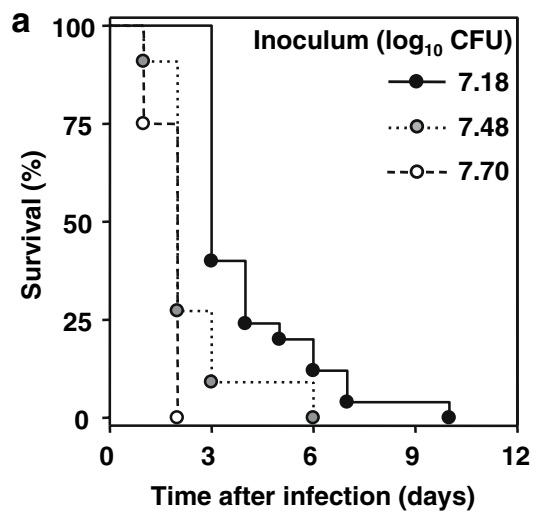

Fig. 1 a Comparison of the survival curves for non-immunosuppressed infant rats infected by intracisternal injection with three different inocula of Aspergillus fumigatus conidia. Survival was inversely proportional to the size of the inoculum. Results of three independent experiments are shown. b Comparison of inoculum size and resulting cerebral fungal burden determined by culture of cortical homogenates

\section{Characterization of the model}

Three independent experiments with groups of $\geq 7$ animals using an inoculum of $7.18 \log _{10} \mathrm{CFU}$ of $A$. fumigatus resulted in survival curves that were not significantly different $(P=\mathrm{ns}$ by log-rank test $)$ with a median survival of 3 days (range 2-10). This similarity of the survival curves validates the reproducibility and robustness of the present model of cerebral aspergillosis (Fig. 2).

To study the kinetics of intracerebral fungal replication during the first 2 days of cerebral Aspergillus infection when animals appear well and death occurs rarely, and to correlate the findings with the phase when mortality occurs, rats were killed $1,2,3$ and 4 days after infection with

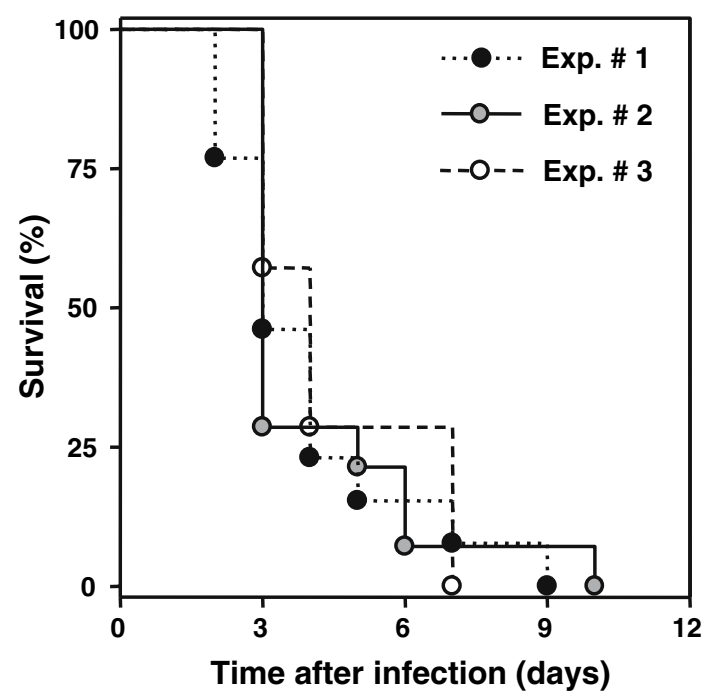

Fig. 2 Comparison of the survival curves from three independent experiments (Exp.) in which infant rats were infected by an intracisternal injection of $7.18 \log _{10}$ CFU of Aspergillus fumigatus conidia. Infection consistently resulted in $100 \%$ mortality within $7-10$ days

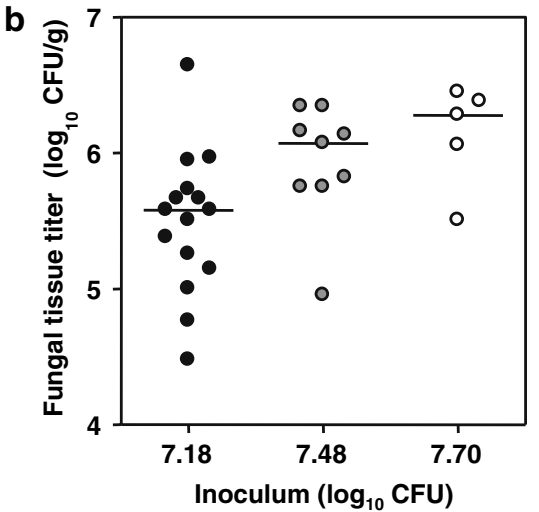

of all animals that died within the first 3 days after infection. The number of CFU of Aspergillus fumigatus recovered from cortical homogenates increases with the size of the inoculum. $(n=15,9$ and 5 for inocula of 7.18, 7.48 and $7.70 \log _{10}$ of colony-forming units, respectively)

$7.18 \log _{10}$ CFU of A. fumigatus ( $n=3$ or 4 per time point), and the cerebral fungal burden was determined by culture. The number of CFU of Aspergillus in cortical homogenates remained stable over the first 4 days after infection at 5.45 (4.30-5.92) $\log _{10}$ CFU/g. In spite of histological evident meningitis, in the majority of animals no cerebrospinal fluid could be aspirated by puncture of the cisterna magna at any time after infection.

Clinical manifestations of cerebral aspergillosis

Tremors starting a few hours after infection and lasting a day (in $84 \%$ of animals) were early signs of disease. Nine of 12 animals with tremors persisting on day 2 died on the next day. In animals infected with $7.18 \log _{10} \mathrm{CFU}$ weight loss starting 2 days prior to death was observed in $52 \%$ of animals. On the day preceding death, $84 \%$ of those animals had lost a mean of $12.5 \%$ of their starting weight. On the same day, the clinical score of all animals infected with an inoculum of $7.18 \log _{10}$ CFU of A. fumigatus was $\geq 4$ (out of a score of 5), indicating that CNS aspergillosis proceeded causing only few signs of disease until the day of death. Circling movements (in $20 \%$ ), limb weakness (in 12\%) and rolling (in 8\%), all indicating CNS disease, were observed shortly before death.

\section{Histopathology}

Fungal meningitis characterized by an infiltrate with predominately polymorphonuclear cells (PMN), conidia, and hyphae was found in $85 \%$ of animals. There were no granulomas. Fungal elements and inflammtory cells were concentrated around meningeal vessels, many of which contained thrombotic material and hyphae that had apparently penetrated the vessel wall (Fig. 3e, f). A median of 6 superficial 
and deep cerebral abscesses (range 2-26) containing abundant fungal hyphae and PMN were documented by histomorphology in all animals (Fig. 3). More abscesses were found in animals dying before day 4 (median 6 (2-26), $n=14)$ than in those dying on or after day $4[2.5(2-5)$ $n=6]$. Abscess location was cerebellar in $48 \%$, periventricular in $41 \%$ and frontal in $11 \%$. No hemorrhages were seen.
Fungal culture

After infection with an inoculum of $7.18 \log _{10} \mathrm{CFU}$ of A. fumigatus, quantitative fungal culture of cortical homogenates yielded maximal growth of 5.58 (4.48-6.64) $\log _{10}$ CFU/g in animals who died on day 3 after infection $(n=15$; Fig. 4a). This fungal burden is similar to the one
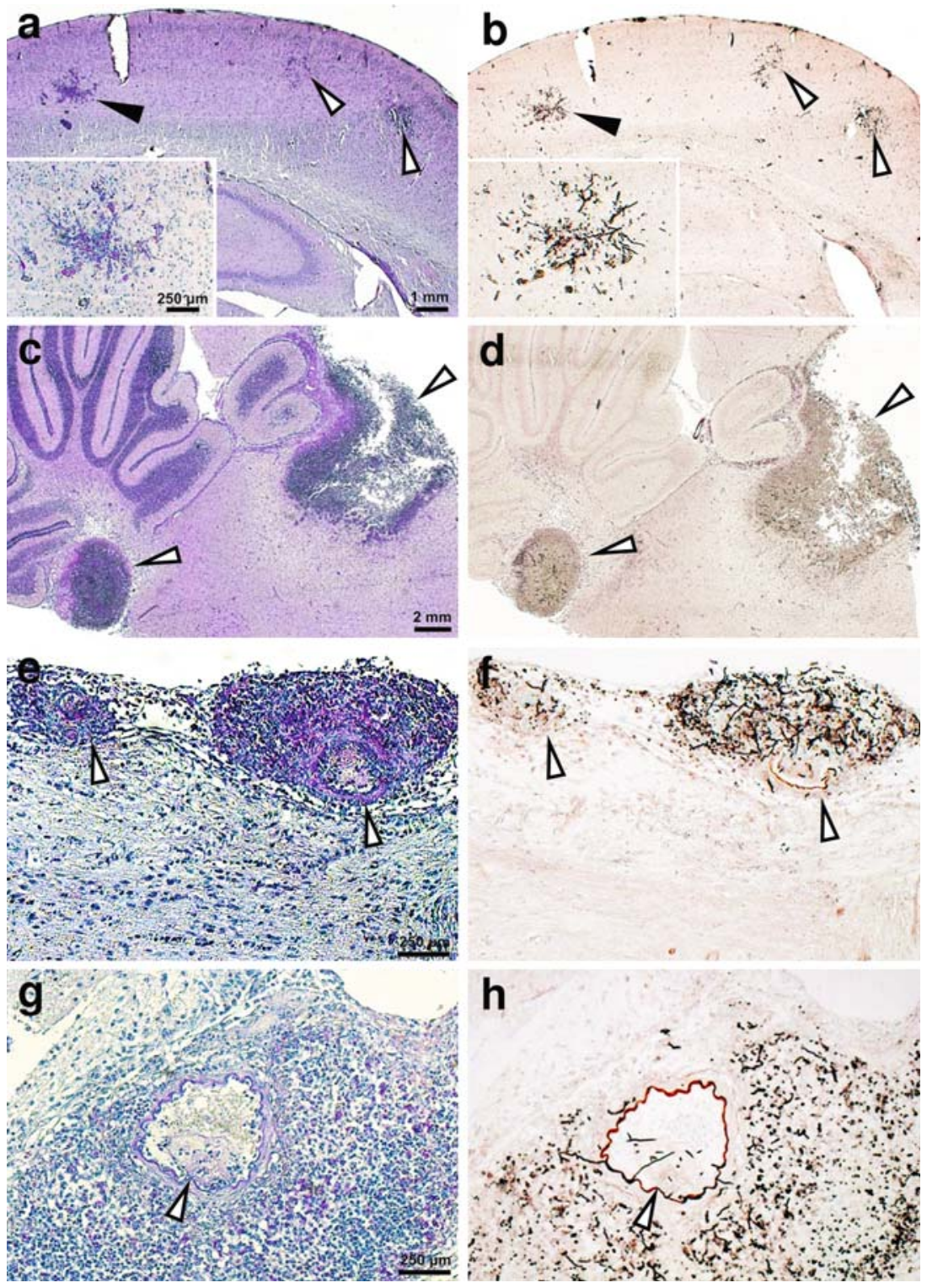

Fig. 3 Histomorphology of animals infected with $7.18 \log _{10}$ CFU of Aspergillus fumigatus. Sections (a), (c), (e) and (g) were stained with periodic-acid Schiff stain; the corresponding adjacent sections (b), (d), (f) and (h) were stained with Grocott's modified Gomori stain. a, b In this animal that died on day 4 after infection, three deep abscesses in the cerebral parenchyma are shown (arrow-heads). The cellular infiltrate is closely associated with fungal hyphae, whose orientation suggests outward growth from a center. The abscess highlighted by the closed arrow-head is enlarged in the insert. The cellular and fungal infiltrate of the subarachnoid space can be seen as a line of dark stain following the contour of the cortical hemisphere. c, $\mathbf{d}$ Two abscesses

(arrow-heads) have formed at the base of the cerebellum in an animal that died on day 4 after infection. Both contain abundant PMNs and fungal elements. e, $\mathbf{f}$ Meningitis characterized by a dense infiltrate of inflammatory cells and fungal elements in the subarachnoid space with involvement of subarachnoid vessels containing thrombotic material (arrow-heads) is shown in this animal that died on day 3 after infection. $\mathbf{g}, \mathbf{h}$ A cerebral artery of a rat that died on day 3 after infection is surrounded by a dense inflammatory infiltrate containing abundant conidia and hyphae. The vessel lumen is obliterated by thrombotic material containing hyphae (arrow-head) 


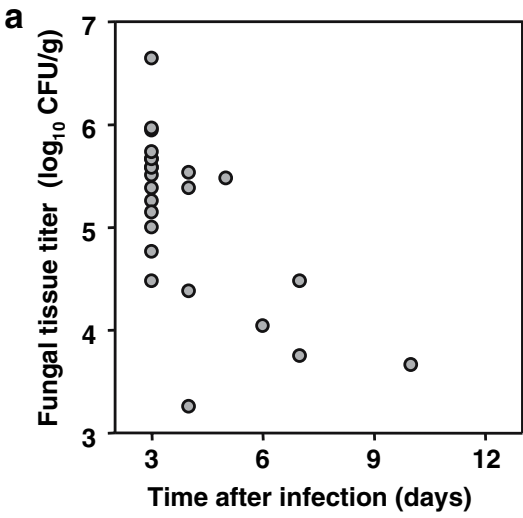

Fig. 4 Quantitation of cerebral fungal burden in animals infected with $7.18 \log _{10}$ CFU of Aspergillus fumigatus. a Fungal culture of all 24 brains yielded growth. Colony-forming unit counts peaked on day 3 at $5.4 \log _{10}$ colony-forming units/g and declined in animals surviving

recovered from animals killed at predefined endpoints irrespective of clinical signs between 1 and 4 days after infection [It was $5.62(4.70-5.92) \log _{10} \mathrm{CFU} / \mathrm{g}$ on day $1 ; 5.44$ (5.26-5.49) $\log _{10} \mathrm{CFU} / \mathrm{g}$ on day 2 ; 5.51 (5.32-5.65) $\log _{10} \mathrm{CFU} / \mathrm{g}$ on day 3 ; and 5.53 (4.30-5.53) $\log _{10} \mathrm{CFU} / \mathrm{g}$ on day 4). Numbers of Aspergillus CFU in cortical homogenates declined over time to a minimum of $3.7 \log _{10} \mathrm{CFU} / \mathrm{g}$ in the only animal that survived for 10 days.

To measure cerebral fungal burden resulting from Aspergillus hyphae, galactomannan concentration in cortical homogenates was determined ( $n=25$; Fig. 4b). Galactomannan is an element of the Aspergillus hyphal cell wall that is absent in conidia. The galactomannan index was highest in animals that died between 3 and 5 days after infection and declined in parallel to the CFU counts in animals that survived beyond 5 days.

No significant correlation was found between CFU counts and galactomannan from 24 samples $(P=0.2$; Person $r=0.26 ; R$ squares $=0.06$ ).

To determine the extent of secondary fungal dissemination from the CNS quantitative fungal culture of visceral organs and the brain was performed. Fungal infection spread to at least one peripheral organ in $83 \%$ of rats. The number of Aspergillus CFU in visceral organs of animals that died on the same day after infection was similar for liver, lung, kidney and spleen, but was two orders of magnitude lower than in the brain. In animals that died on day 3 $(n=11)$ after infection with $7.18 \log _{10}$ CFU of A. fumigatus, all brains were culture positive, all animals had positive cultures from at least one visceral organ, but only two animals $(18 \%)$ had positive fungal cultures from all visceral organs (Fig. 5).

Similar to the brain, fungal titers in visceral organs declined over time. Three of 19 animals (that died on days 4 , 6 and 7) had sterile cultures from all organs except the brain, suggesting spontaneous clearance of systemic infection.

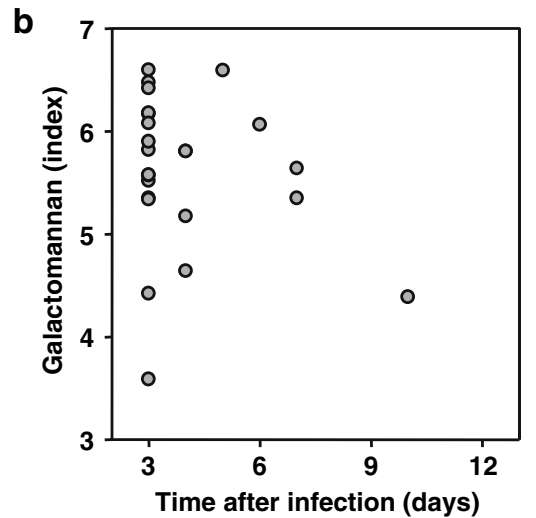

longer. b The galactomannan index was highest in animals that died $3-$ 5 days after infection and declined parallel to the colony-forming units in animals surviving $>5$ days

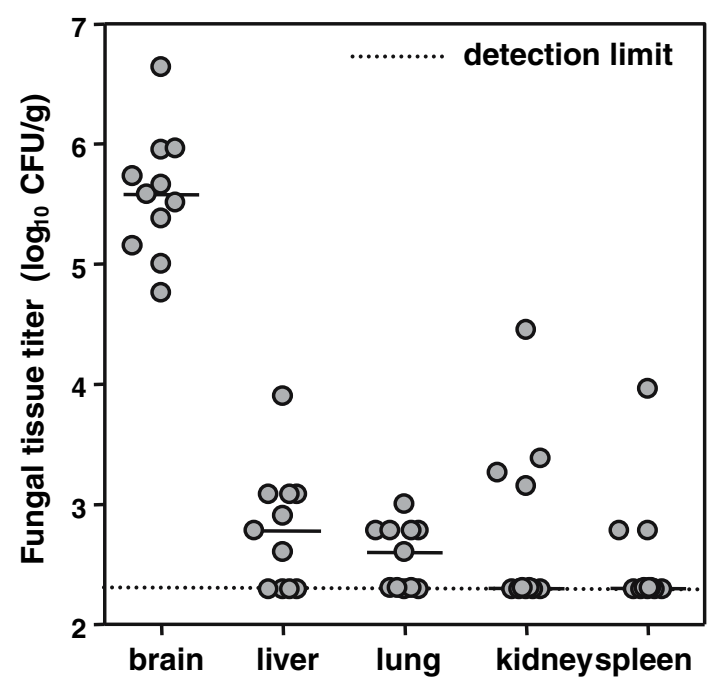

Fig. 5 Fungal titers of selected organs from animals that died on day 3 after intracisternal infection with $7.18 \log _{10}$ CFU of Aspergillus fumigatus $(n=11)$. Negative organ cultures were set to the detection limit at $2.29 \log _{10}$

\section{Discussion}

The development of a model of CNS aspergillosis in animals without the use of iatrogenic immunosuppression provides the opportunity to study the pathogenesis of this disease, the host response and treatment regimens. All the criteria established for a successful animal model of aspergillosis are fulfilled [9]: disease parameters such as cerebral fungal burden and mortality can be determined by the size of the fungal inoculum at infection. Mortality most likely results from cerebral aspergillosis since systemic dissemination of fungi is controlled by the host response. The model is robust and reproducible. Infant rats with their dams are unproblematic and economical to house. In the 
absence of immunosuppression, sterile animal facilities are not needed to protect the animals from opportunistic infections. Injection of the fungal inoculum into the cisterna magna caused meningitis involving predominantly PMNs. The infiltrates with the highest density of fungal elements and PMNs were found around cerebral vessels, many of which were obliterated by thrombi containing fungal hyphae, demonstrating both the angiotropism and propensity of Aspergillus fumigatus to penetrate vessel walls. The cerebellar and periventricular location of the majority of fungal abscesses may reflect the postero-anterior concentration gradient resulting from injection of the infectious conidia in the cisterna magna. Deep abscesses where the radial distribution of hyphae suggested outward growth from a center may have originated from hematogenously disseminated fungal elements or from conidia in the VirchowRobbins space of cerebral vessels.

Intracisternal injection of the fungal inoculum mimics the route of human CNS infection in acute and chronic invasive Aspergillus sinusitis [13, 19, 21]. In this setting, meningeal infiltration with inflammatory cells and hyphae is associated with arteritis and vascular thrombosis resulting in ischemic or hemorrhagic necrosis and abscess formation, similar to the histopathologic findings in the present model. Most cases of human cerebral aspergillosis result from hematogenous dissemination of fungal elements from foci of invasive disease most commonly in the lungs. CNS aspergillosis is characterized by invasion of vascular walls by hyphae resulting in thrombosis and ischemic infarcts, where fungi remain almost entirely within the vessels, hemorrhagic infarcts and abscesses [2, 16, 18, 30]. In an autopsy series of 45 patients with CNS aspergillosis, meningitis adjacent to leptomeningeal vessels containing fungal thrombi was found in virtually all patients [18]. PMNs were the dominant inflammatory cells present. In organ transplant recipients with CNS aspergillosis, meningitis was found in $10 \%$ of patients in association with necrotizing cortical lesions [30].

Abscesses composed of a core of necrotic tissue surrounded by an area of hemorrhagic necrosis, inflammatory cells and hyphae were found in all patients autopsied [18]. Human CNS aspergillosis is well mimicked by our model with respect to histopathology, rapid disease progression and universal mortality in the absence of treatment.

In the present model, the time of death was related to severity of disease: animals dying by day 3 after infection had higher cerebral fungal concentrations determined by culture, higher galactomannan levels and a higher number of cerebral abscesses than animals dying at later time points. While both hyphae and non-germinated conidia are measured by culture, galactomannan determination is specific for hyphae, which may explain why there was no significant correlation between the two measures of fungal burden. Another factor may be underscoring the $\mathrm{CFU}$ due to large colonies.

Most rodent models of invasive aspergillosis require immunosuppression to produce consistent disease [12, 17, $20,25,26]$. Immunocompetent mice can be infected by the i.v. route, but they tend to clear the infection from lung, liver and spleen, and often from the brains, and die with high fungal concentrations in their kidneys [11]. Intracerebral injection of conidia in non-immunosuppressed mice either resulted in $100 \%$ lethality in the first 2 days or survival of more than $50 \%$ of the animals, depending on the size of the inoculum that could not be more finely titrated [3]. The model presented here is, to our knowledge, the first in which consistent cerebral infection is produced in rodents that are not iatrogenically immunosuppressed or selected for genetic defects in their immune response. It is generally accepted that nursing rats between 11 and 21 days of age are immunologically less competent than adult animals. However, it is largely unknown in what respects exactly an infant rat's immature immune system differs from that of an adult animal and to what extent the infant rats' immune system becomes more competent as the animals mature to adolescence.

Infection spreads from the CNS to other organs, but fungal concentrations are two to three orders of magnitude lower than in the brain and some animals cleared peripheral infection as early as by day 4 after infection. A potential advantage of an animal model in a non-immunosuppressed host is that efficient treatments may prove curative, an obvious goal in the patient and one difficult to achieve in immunocompromised animals [7]. In addition, the absence of iatrogenic immunosuppression may be beneficial for studies of the pathophysiology of aspergillosis. However, cerebral aspergillosis in humans most commonly occurs in patients with at least temporary neutropenia or functionally impaired neutrophils, which highlights a limitation of the present model. Pharmacokinetics and pharmacodynamics of many drugs, including antifungals, differ between infants and adults, emphasizing the necessity to monitor drug concentrations in serum and target organ when performing treatment studies with our model.

Compared to the model of cerebral aspergillosis that uses intracerebral injection of conidia to induce cerebral abscesses in immunocompromised mice, our model has several advantages: intracisternal infection is minimally traumatic to the animals, is well tolerated (no deaths related to the procedure occurred) and does not induce structural brain damage. Systemic dissemination is at a very low level compared to the cerebral fungal burden, is controlled by the host, and does not appear to contribute to morbidity and mortality.

This model should be useful for the study of host-pathogen interactions and disease mechanisms, and the evaluation of novel therapeutic approaches to cerebral aspergillosis. 
Acknowledgments We thank Angela Bühlmann and Jürg Kummer for their excellent technical assistance, Martin G. Täuber for critical reading of the manuscript and Bio-Rad Laboratories AG, Reinach for supplying Platelia Aspergillus test kits.

\section{References}

1. Bifrare YD, Kummer J, Joss P, Täuber MG, Leib SL (2005) Brainderived neurotrophic factor protects against multiple forms of brain injury in bacterial meningitis. J Infect Dis 191:40-45

2. Boes B, Bashir R, Boes C, Hahn F, McConnell JR, McComb R (1994) Central nervous system aspergillosis: analysis of 26 patients. J Neuroimaging 4:123-129

3. Chiller TM, Luque JC, Sobel RA, Farrokhshad K, Clemons KV, Stevens DA (2002) Development of a murine model of cerebral aspergillosis. J Infect Dis 186:574-577

4. Chiller TM, Sobel RA, Luque JC, Clemons KV, Stevens DA (2003) Efficacy of amphotericin B or itraconazole in a murine model of central nervous system Aspergillus infection. Antimicrob Agents Chemother 47:813-815

5. Clemons KV, Espiritu M, Parmar R, Stevens DA (2005) Comparative efficacies of conventional amphotericin $\mathrm{b}$, liposomal amphotericin B (AmBisome), caspofungin, micafungin, and voriconazole alone and in combination against experimental murine central nervous system aspergillosis. Antimicrob Agents Chemother 49:4867-4875

6. Clemons KV, Parmar R, Martinez M, Stevens DA (2006) Efficacy of Abelcet alone, or in combination therapy, against experimental central nervous system aspergillosis. J Antimicrob Chemother 58:466-469

7. Clemons KV, Stevens DA (2006) Animal models of Aspergillus infection in preclinical trials, diagnostics and pharmacodynamics: what can we learn from them? Med Mycol 44:119-126

8. Clemons KV, Stevens DA (2004) Comparative efficacies of four amphotericin B formulations-Fungizone, amphotec (Amphocil), AmBisome, and Abelcet-against systemic murine aspergillosis. Antimicrob Agents Chemother 48:1047-1050

9. Clemons KV, Stevens DA (2005) The contribution of animal models of aspergillosis to understanding pathogenesis, therapy and virulence. Med Mycol 43(Suppl 1):S101-S110

10. Coimbra RS, Voisin V, de Saizieu AB, Lindberg RL, Wittwer M, Leppert D, Leib SL (2006) Gene expression in cortex and hippocampus during acute pneumococcal meningitis. BMC Biol 4:15

11. Denning DW, Stevens DA (1991) Efficacy of cilofungin alone and in combination with amphotericin B in a murine model of disseminated aspergillosis. Antimicrob Agents Chemother 35:1329-1333

12. Dixon DM, Polak A, Walsh TJ (1989) Fungus dose-dependent primary pulmonary aspergillosis in immunosuppressed mice. Infect Immun 57:1452-1456

13. Hope WW, Walsh TJ, Denning DW (2005) The invasive and saprophytic syndromes due to Aspergillus spp. Med Mycol 43(Suppl 1):S207-S238

14. Imai J, Singh G, Fernandez B, Clemons KV, Stevens DA (2005) Efficacy of Abelcet and caspofungin, alone or in combination, against CNS aspergillosis in a murine model. J Antimicrob Chemother 56:166-171

15. Imai JK, Singh G, Clemons KV, Stevens DA (2004) Efficacy of posaconazole in a murine model of central nervous system aspergillosis. Antimicrob Agents Chemother 48:4063-4066

16. Jantunen E, Volin L, Salonen O, Piilonen A, Parkkali T, Anttila VJ, Paetau A, Ruutu T (2003) Central nervous system aspergillosis in allogeneic stem cell transplant recipients. Bone Marrow Transplant 31:191-196

17. Kirkpatrick WR, Perea S, Coco BJ, Patterson TF (2002) Efficacy of caspofungin alone and in combination with voriconazole in a guinea pig model of invasive aspergillosis. Antimicrob Agents Chemother 46:2564-2568

18. Kleinschmidt-DeMasters BK (2002) Central nervous system aspergillosis: a 20-year retrospective series. Hum Pathol 33:116-124

19. Lammens M, Robberecht W, Waer M, Carton H, Dom R (1992) Purulent meningitis due to aspergillosis in a patient with systemic lupus erythematosus. Clin Neurol Neurosurg 94:39-43

20. Latgé JP (1999) Aspergillus fumigatus and aspergillosis. Clin Microbiol Rev 12:310-350

21. Lowe J, Bradley J (1986) Cerebral and orbital Aspergillus infection due to invasive aspergillosis of ethmoid sinus. J Clin Pathol 39:774-778

22. Meli DN, Coimbra RS, Erhart DG, Loquet G, Bellac CL, Täuber MG, Neumann U, Leib SL (2006) Doxycycline reduces mortality and injury to the brain and cochlea in experimental pneumococcal meningitis. Infect Immun 74:3890-3896

23. Meli DN, Loeffler JM, Baumann P, Neumann U, Buhl T, Leppert D, Leib SL (2004) In pneumococcal meningitis a novel watersoluble inhibitor of matrix metalloproteinases and TNF-alpha converting enzyme attenuates seizures and injury of the cerebral cortex. J Neuroimmunol 151:6-11

24. Pagano L, Ricci P, Montillo M, Cenacchi A, Nosari A, Tonso A, Cudillo L, Chierichini A, Savignano C, Buelli M, Melillo L, La Barbera EO, Sica S, Hohaus S, Bonini A, Bucaneve G, Del Favero A (1996) Localization of aspergillosis to the central nervous system among patients with acute leukemia: report of 14 cases. Gruppo Italiano Malattie Ematologiche dell'Adulto Infection Program. Clin Infect Dis 23:628-630

25. Petraitiene R, Petraitis V, Groll AH, Sein T, Piscitelli S, Candelario M, Field-Ridley A, Avila N, Bacher J, Walsh TJ (2001) Antifungal activity and pharmacokinetics of posaconazole $(\mathrm{SCH}$ 56592 ) in treatment and prevention of experimental invasive pulmonary aspergillosis: correlation with galactomannan antigenemia. Antimicrob Agents Chemother 45:857-869

26. Ruijgrok EJ, Fens MH, Bakker-Woudenberg IA, van Etten EW, Vulto AG (2005) Nebulization of four commercially available amphotericin B formulations in persistently granulocytopenic rats with invasive pulmonary aspergillosis: evidence for long-term biological activity. J Pharm Pharmacol 57:1289-1295

27. Schwartz S, Ruhnke M, Ribaud P, Corey L, Driscoll T, Cornely OA, Schuler U, Lutsar I, Troke P, Thiel E (2005) Improved outcome in central nervous system aspergillosis, using voriconazole treatment. Blood 106:2641-2645

28. Sellner J, Leib SL (2006) In bacterial meningitis cortical brain damage is associated with changes in parenchymal MMP-9/TIMP1 ratio and increased collagen type IV degradation. Neurobiol Dis 21:647-656

29. Singh G, Imai J, Clemons KV, Stevens DA (2005) Efficacy of caspofungin against central nervous system Aspergillus fumigatus infection in mice determined by TaqMan PCR and CFU methods. Antimicrob Agents Chemother 49:1369-1376

30. Torre-Cisneros J, Lopez OL, Kusne S, Martinez AJ, Starzl TE, Simmons RL, Martin M (1993) CNS aspergillosis in organ transplantation: a clinicopathological study. J Neurol Neurosurg Psychiatry 56:188-193

31. Wasylnka JA, Moore MM (2002) Uptake of Aspergillus fumigatus conidia by phagocytic and nonphagocytic cells in vitro: quantitation using strains expressing green fluorescent protein. Infect Immunol 70:3156-3163 